\title{
Using conditioned suppression to investigate compulsive drug seeking in rats
}

\author{
Jules H.W. Limpens ${ }^{a}$, Evelien H.S. Schut ${ }^{a}$, Pieter Voorn ${ }^{b}$, Louk J.M.J. Vanderschuren ${ }^{\mathrm{a}, \mathrm{c}, *}$ \\ a Brain Center Rudolf Magnus, Department of Translational Neuroscience, University Medical Center Utrecht, Utrecht, The Netherlands \\ ${ }^{\mathrm{b}}$ Department of Anatomy and Neurosciences, Neuroscience Campus Amsterdam, VU University Medical Centre, Amsterdam, The Netherlands \\ ' Department of Animals in Science and Society, Division of Behavioural Neuroscience, Faculty of Veterinary Medicine, Utrecht University, Utrecht, The \\ Netherlands
}

\section{A R T I C L E I N F O}

\section{Article history:}

Received 17 April 2014

Received in revised form 13 June 2014

Accepted 28 June 2014

Available online 8 July 2014

\section{Keywords:}

Cocaine

Sucrose

Self-administration

Conditioned suppression

Compulsive behaviour

\begin{abstract}
A B S T R A C T
Background: Persistent drug seeking despite harmful consequences is a defining characteristic of addiction. Recent preclinical studies have demonstrated the occurrence of this hallmark feature of addictive behaviour in rodents. For example, it has been shown that the ability of an aversive conditioned stimulus (CS) to suppress cocaine seeking was diminished after an extended self-administration history. The present study aimed to optimize the experimental conditions to examine conditioned suppression of sucrose and cocaine seeking in rats, and its dependence on the longevity of self-administration experience.

Methods: We investigated whether conditioned suppression depends on the intensity and quantity of footshocks during conditioning. In addition, the effects of CS omission, extinction and reconditioning were investigated, as well as the influence of the CS interval sequence on conditioned suppression. We also compared conditioned suppression after a limited and extended sucrose or cocaine self-administration history.

Results: We found that conditioned suppression depended on the intensity rather than the quantity of footshocks, whereby a higher footshock intensity was necessary to induce suppression of cocaine seeking compared to sucrose seeking. Conditioned suppression was most pronounced when the test started with presentation of the aversive CS, and conditioned suppression could be extinguished and reacquired. In addition, conditioned suppression of cocaine, but not sucrose seeking was reduced after extended self-administration experience.

Conclusions: These data provide a detailed analysis of conditioned suppression of cocaine and sucrose seeking. Importantly, we confirm the usefulness of conditioned suppression to study persistent drug seeking after prolonged drug self-administration.
\end{abstract}

(c) 2014 Elsevier Ireland Ltd. All rights reserved.

\section{Introduction}

Drug addiction is a chronic relapsing brain disorder, characterized by persistent drug-directed behaviour even with explicit knowledge of its negative consequences (American Psychiatric Association, 2000, 2013; Leshner, 1997; O’Brien and McLellan, 1996; Volkow and Li, 2004). It has been estimated that 27 million people worldwide are addicted to illicit drugs, and 76 million suffer from alcohol use disorder (United Nations Office on Drugs and

\footnotetext{
* Corresponding author at: Department of Animals in Science and Society, Division of Behavioural Neuroscience, Faculty of Veterinary Medicine, Utrecht University, Utrecht, The Netherlands. Tel.: +31302535239.

E-mail address: 1.j.m.j.vanderschuren@uu.nl (L.J.M.J. Vanderschuren).
}

Crime, 2012; World Health Organization, 2004). Pharmacotherapies to treat addiction are limited in number and efficacy (Koob et al., 2009; O'Brien, 2008; Pierce et al., 2012; van den Brink, 2012). Therefore, a formidable challenge for addiction research is to unravel the neural mechanisms underlying addictive behaviour, in order to facilitate the development of novel treatments for this devastating disorder. In order to achieve this, it is essential to model the core features of addictive behaviour in animals.

Based on this idea, a number of animal studies have attempted to emulate aspects of genuine addiction-like behaviour, such as escalation of drug use, resistance to extinction, increased motivation for drugs and drug seeking despite adverse consequences (for reviews see Lesscher and Vanderschuren, 2012; Vanderschuren and Ahmed, 2013). Studies that examine continued drug use despite adverse consequences often employ punishment paradigms. For example, 
adulterating alcohol with the bitter tastant quinine is used as a punishment method in studies of alcohol addiction (Hopf et al., 2010; Lesscher et al., 2010; Wolffgramm, 1991). In other work, interoceptive aversion (e.g., histamine), post-ingestion illness (e.g., lithium chloride), or footshocks or shock-associated stimuli to punish drug seeking or taking are used (Deroche-Gamonet et al., 2004; Dickinson et al., 2002; Freeman et al., 2014; Kearns et al., 2002; Pelloux et al., 2007; Vanderschuren and Everitt, 2004). Recently, it has been shown that the ability of footshocks or shock-associated stimuli to suppress cocaine seeking is diminished after an extended cocaine self-administration history (DerocheGamonet et al., 2004; Jonkman et al., 2012; Pelloux et al., 2007; Vanderschuren and Everitt, 2004). Such persistent drug seeking behaviour after prolonged cocaine self-administration is thought to reflect the unflagging pursuit of drugs observed in human addicts (American Psychiatric Association, 2000, 2013; Leshner, 1997; O'Brien and McLellan, 1996; Volkow and Li, 2004). Therefore, we think that conditioned suppression of drug seeking is a useful way to study drug seeking behaviour despite adverse consequences. However, the conditions under which conditioned suppression of drug seeking can be observed remain incompletely understood.

To enhance our understanding of persistent drug seeking, the first aim of the present study was to provide an extensive parametric characterization of conditioned suppression of cocaine and sucrose seeking. To this end, we examined the effect of different footshock intensities and quantities during the acquisition of the conditioned stimulus (CS)-footshock association on conditioned suppression of cocaine and sucrose seeking. In addition, we studied the role of the CS-sequence presentation, CS extinction and re-acquisition. The second aim was to use the optimal experimental parameters to examine the extent to which the aversive CS suppressed seeking behaviour in rats with a limited or extended sucrose or cocaine self-administration history, respectively.

\section{Methods}

\subsection{Animals}

Male Wistar rats (Charles River, Sulzfeld, Germany) weighing 260-280 g at the time of arrival were individually housed in Macrolon cages $(40 \times 25 \times 18 \mathrm{~cm}$; $l \times w \times h$ ) in climate-controlled rooms (temperature $20-21^{\circ} \mathrm{C}, 55 \pm 15 \%$ relative humidity) under a reversed $12 \mathrm{~h}$ light-dark cycle (lights on at $19.00 \mathrm{~h}$ ). Animals were allowed to habituate to the housing conditions for at least 9 days before surgery. Rats received $20 \mathrm{~g}$ chow (SDS) per day, which is sufficient to maintain body weight and growth. Water was available ad libitum. Self-administration sessions were carried out between 9 am and $6 \mathrm{pm}$, for 5-7 days a week. Experiments were approved by the Animal Ethics Committee of Utrecht University, and were conducted in agreement with Dutch legislation (Wet op de dierproeven, 1996) and European regulations (Guideline 86/609/EEC).

\subsection{Apparatus}

All subjects were trained and tested in operant conditioning chambers $(29.5 \times 24 \times 25 \mathrm{~cm} ; l \times w \times h$; Med Associates, Georgia, VT, USA). The chambers were placed in light- and sound-attenuating cubicles equipped with a ventilation fan. Each chamber was equipped with two $4.8 \mathrm{~cm}$ wide retractable levers, placed $11.7 \mathrm{~cm}$ apart and $6.0 \mathrm{~cm}$ from the grid floor. The assignment of the left and right lever as seeking and taking lever (see below) was counterbalanced across rats. A cue light $(28 \mathrm{~V}, 100 \mathrm{~mA})$ was present above each lever and a house light $(28 \mathrm{~V}, 100 \mathrm{~mA})$ was located on the opposite wall. A liquid dipper $(0.04 \mathrm{ml})$ delivered sucrose solution to a recessed magazine situated between the levers. Cocaine infusions were controlled by a syringe pump placed on top of the cubicles. During the cocaine self-administration sessions, polyethylene tubing ran from the syringe placed in the syringe pump via a swivel to the cannula on the subjects' back; in the operant chamber tubing was shielded with a metal spring. Experimental events and data recording were controlled by procedures written in MedState Notation using MED-PC for Windows.

\subsection{Surgery}

Rats allocated to cocaine self-administration experiments were anaesthetised with ketamine hydrochloride (Narketan, $75 \mathrm{mg} / \mathrm{kg}$, i.m.) and medetomidine hydrochloride (Cepetor, $0.4 \mathrm{mg} / \mathrm{kg}$, s.c.) and supplemented with ketamine as needed.
A single intravenous catheter was implanted into the right jugular vein aimed at the left vena cava. Catheters (Camcaths, Cambridge, UK) consisted of a $22 \mathrm{~g}$ cannula attached to silastic tubing (0.012 ID) and fixed to nylon mesh. The mesh end of the catheter was sutured subcutaneously (s.c.) on the dorsum. Carprofen $(50 \mathrm{mg} / \mathrm{kg}$, s.c.) was administrated once before and twice after surgery. Gentamycin $(5 \mathrm{mg} / \mathrm{kg}$, s.c.) was administered before surgery and for 5 days post-surgery. Animals were allowed 7-9 days to recover from surgery.

\subsection{Behavioural procedures}

2.4.1. Cocaine self-administration. Rats were trained to lever press for cocaine under a heterogeneous seeking-taking (ST) chain schedule of reinforcement (Olmstead et al., 2000; Vanderschuren and Everitt, 2004; Veeneman et al., 2012) with a random interval (RI) of $120 \mathrm{~s}$ on the seeking link (ST(RI-120)). Self-administration training started with the acquisition of the taking response under a fixed-ratio 1 (FR-1) schedule of reinforcement. During acquisition sessions, only the taking lever was present. Pressing this lever resulted in the infusion of $0.25 \mathrm{mg}$ cocaine in $0.1 \mathrm{ml}$ saline delivered over $5.6 \mathrm{~s}$, the illumination of the cue light above the taking lever for $5.6 \mathrm{~s}$, the retraction of the lever and the switching off of the house light. After a $20 \mathrm{~s}$ time-out period, the taking lever was reintroduced and the house light illuminated, signalling the start of a new cycle. Once animals had acquired cocaine self-administration, they were gradually introduced to the ST chain schedule, starting with a schedule with a RI requirement of $2 \mathrm{~s}$ on the seeking link. ST(RI)-sessions started with the introduction of the seeking lever and the illumination of the house light. The first press on the seeking lever initiated the RI and pressing this lever was without consequences until the RI had elapsed. When the RI had elapsed, pressing the seeking lever resulted in retraction of the seeking lever and insertion of the taking lever. Next, responding on the taking lever (under the FR-1 schedule of reinforcement) resulted in an infusion with cocaine, illumination of the cue light, retraction of the taking lever and the switching off of the house-light. This was followed by a $10 \mathrm{~min}$ time-out period to minimize the influence of cocaine-induced psychomotor effects on responding for the next infusion. After the time-out period, a new cycle started with the reintroduction of the seeking lever and the illumination of the house-light. When the rats had acquired the task under a RI of $2 \mathrm{~s}$, the RI was progressively increased between sessions until animals had acquired the task under an RI of $120 \mathrm{~s}$. The program automatically ended after $2 \mathrm{~h}$ or if animals had obtained 10 rewards, whichever occurred first. After each self-administration session, intravenous catheters were flushed with a gentamycin-heparin-saline solution to maintain the patency of the catheters. Priming infusions of cocaine to stimulate self-administration were never given

2.4.2. Sucrose self-administration. Rats were trained to lever press for sucrose under a ST(RI-120) schedule of reinforcement. This procedure was similar to the ST(RI-120) with cocaine as the reward, with the following exceptions. After a response on the taking lever, $0.2 \mathrm{ml}$ of a $20 \%$ sucrose solution was delivered by presenting the dipper five times for $5 \mathrm{~s}$ at a rate of one presentation per second. In addition, the session was terminated when $30 \mathrm{~min}$ had passed or when a maximum of 30 rewards had been obtained during the FR- 1 training, or after 10 rewards during the RI training sessions.

2.4.3. Acquisition of the CS-shock association. Once stable responding under the ST(RI-120) schedule was achieved (i.e. when the mean number of seeking responses per minute of the last three training sessions of an individual rat did not exceed a difference of $10 \%$ of the overall mean of those three sessions), rats were assigned to groups that either underwent conditioning with CS-footshock pairings (CS-shock group) or underwent control conditioning (control group). Assignment to the groups was based on the mean seeking responses per minute and seeking latency of the three last training sessions prior to conditioning, so that CS-shock and control groups had equal mean response rates and seeking latencies.

Acquisition of the CS-shock association was established in operant chambers different from those where the rats had received training for self-administration of sucrose or cocaine. To facilitate CS-shock, rather than context-shock association, the animals were pre-exposed to the shock boxes for $30 \mathrm{~min}$ for 2 days. The CS-shock conditioning session comprised a lead-in period of $5 \mathrm{~min}$ followed by two periods of $10 \mathrm{~min}$ with a $85 \mathrm{~dB}, 2900 \mathrm{~Hz}$ tone (with an intertrial-interval of $10 \mathrm{~min}$ ) during which 10 unpredictable, scrambled footshocks ( 1 s duration) were delivered (i.e., 20 shocks in total). The tone-shock association session ended with a lead-out period of $5 \mathrm{~min}$. Rats in the control group were subjected to the same procedure but without the delivery of footshocks.

2.4.4. Conditioned suppression of sucrose- and cocaine-seeking behaviour. After conditioning, rats received 4 additional ST(RI-120) training sessions. Subsequently, a test session for conditioned suppression of sucrose- or cocaine-seeking behaviour was performed. This conditioned suppression test was conducted in the same operant chambers where rats received self-administration training. After a lead-in period of $2 \mathrm{~min}$, the seeking lever was inserted for $14 \mathrm{~min}$ with the house light illuminated. Two-minute intervals in which the tone CS was presented (CS-ON interval) were alternated with two-minute intervals where the tone CS was absent (CS-OFF interval). 


\subsection{Experiments}

2.5.1. Experiment 1: optimization of the experimental conditions for conditioned suppression of sucrose and cocaine seeking. In experiment $1 \mathrm{~A}$, the effect of footshock intensity on conditioned suppression of sucrose and cocaine-seeking behaviour was investigated. To that aim, animals in the CS-shock group underwent fear conditioning with 20 footshocks with shock intensities ranging from $0.25 \mathrm{~mA}$ to $0.45 \mathrm{~mA}$. Five sub-experiments were performed, to test (1) 0.25 and $0.30 \mathrm{~mA}$ shocks; (2) 0.35 and $0.45 \mathrm{~mA}$ shocks; (3) $0.40 \mathrm{~mA}$ shocks in sucrose self-administering animals and (4) $0.35 \mathrm{~mA}$ shocks; (5) 0.40 and $0.45 \mathrm{~mA}$ shocks in cocaine self-administering animals. Since the data in the respective control groups did not differ, these experiments were collapsed for statistical analysis.

Since we found substantial conditioned suppression of seeking behaviour in animals that underwent CS-shock conditioning with 20 footshocks, the effect of footshock quantity on conditioned suppression of sucrose seeking was investigated in experiment $1 \mathrm{~B}$. Hence, animals that were assigned to a CS-shock group received 10 or 20 footshocks. The CS-shock association session of the 20 footshocksgroup existed of a lead-in period of $5 \mathrm{~min}$, after which the rats were presented twice with a $85 \mathrm{~dB}, 2900 \mathrm{~Hz}$ tone for $10 \mathrm{~min}$ (with an intertrial-interval of $10 \mathrm{~min}$ ) during which 10 unpredictable, scrambled footshocks were delivered ( 0.35 or $0.40 \mathrm{~mA}$ ). The tone-shock association session ended with a lead-out period of $5 \mathrm{~min}$. Rats that were assigned to the 10 footshocks-group received only one $10 \mathrm{~min}$ tone presentation, during which 10 unpredictable footshocks were delivered.

To assess the influence of differences in basal levels of responding on conditioned suppression of sucrose seeking, we examined the effect of different CS interval sequences in experiment $1 \mathrm{C}$. Hence, for animals assigned to the CS-OFF group, the test session started with a 2 min period during which no CS was presented. For animals assigned to the CS-ON group, the test session started with a 2 min period during which the CS was presented. Animals in the CS-ON and CS-OFF groups were subjected to CS-shock conditioning with 20 footshocks of $0.35 \mathrm{~mA}$, as above.

In order to test the feasibility of assessing conditioned suppression withinsubjects, we investigated whether conditioned suppression of sucrose seeking could be extinguished and re-acquired in experiment 1D. To that aim, after the animals underwent CS-shock (20 footshocks, $0.35 \mathrm{~mA}$ ) or control conditioning, responding on the seeking lever was first measured during a no CS-session. This session was similar to a conditioned suppression test, but without the presence of the aversive CS. Next, behavioural responding was analysed during a conditioned suppression test with the presence of the aversive CS. Subsequently, animals underwent extinction of the aversive CS in 5 daily sessions. These sessions were similar to the CS-shock conditioning session but without delivery of footshocks. Behavioural responding was then analysed during a third conditioned suppression test. Next, the animals underwent one more CS-shock conditioning session (to reinstate the CS-footshock association) followed by another (fourth) conditioned suppression test. Thus, in experiment 1D, behavioural responding was analysed four times: (1) a no CS-session, (2) a conditioned suppression session, (3) after 5 extinction sessions and (4) after CS-shock reconditioning.
2.5.2. Experiment 2: effect of prolonged self-administration experience on conditioned suppression of sucrose or cocaine-seeking behaviour. This experiment determined the effect of prolonged self-administration experience on conditioned suppression of sucrose and cocaine-seeking behaviour. See Fig. 1 for an outline of the behavioura procedures. Animals assigned to the limited experience group underwent CS-shock conditioning and testing for conditioned suppression once stable responding under the ST(RI-120) schedule was achieved (as described above). Animals assigned to the prolonged experience group were trained for an additional 40 FR-1 daily training sessions once stable responding under the ST(RI-120) schedule was achieved. These FR- 1 cocaine self-administration sessions lasted for $3 \mathrm{~h}$, during which the rats earned an average number of 45 cocaine infusions. To keep the number of obtained rewards between cocaine and sucrose similar, animals assigned to the prolonged sucrose group were therefore restricted to a maximum of 45 reinforcements. Next, animals were assigned to groups that either underwent conditioning with CS-footshock pairings (CS-shock group) or underwent control conditioning (control group). Assignment was based on the mean seeking responses per minute and seeking latency of the three last training sessions prior to conditioning, so that CS-shock and control groups had equal mean response rates and seeking latencies. Acquisition of the CS-shock association and testing for conditioned suppression was performed with optimal experimental conditions as obtained in experiment 1 : (1) a footshock intensity of $0.35 \mathrm{~mA}$ (sucrose) or $0.40 \mathrm{~mA}$ (cocaine). (2) CS association session with 20 footshocks. (3) Start the conditioned suppression test with CS-ON interval (see above for a detailed description of the CS-shock conditioning session and the conditioned suppression test). To investigate whether the incentive value of the reward changed over an extended self-administration period a comparison was made of the average response rate of the last three sessions under the ST(RI-120) schedule after limited and extended cocaine and sucrose self-administration.

\subsection{Statistical analysis}

The following parameters were analysed to test for conditioned suppression: (1) Suppression ratio, which was calculated as [(number of responses during CS-OFF - number of responses during CS-ON)/(number of responses during CS$\mathrm{OFF}+$ number of responses during $\mathrm{CS}-\mathrm{ON})]$. A suppression ratio of 0 or lower means no suppression, a suppression ratio of 1 means complete suppression of responding during CS-ON periods. (2) The latency to make the first response on the seeking lever. Main effects were analysed with a Kruskal-Wallis $H$ test followed by a posthoc Mann-Whitney $U$ test when appropriate. In experiment 1D, main effects of the aversive stimuli on suppression ratio and latency to first response were first analysed with a Friedman test, followed by a post-hoc Mann-Whitney $U$ test. In experiment $1 \mathrm{C}$, the effects of the aversive stimuli on the number of responses during the conditioned suppression test were analysed using ANOVA with group (Contro vs. CS-shock) as between-subjects factor and CS (CS-ON vs. CS-OFF) and time (1st vs. 2nd period) as within-subjects factor, followed by post-hoc $t$-tests when appropriate. In experiment 2 , the effects of self-administration history on baseline response rates under the ST(RI-120) schedule of reinforcement were analysed with a $t$-test.

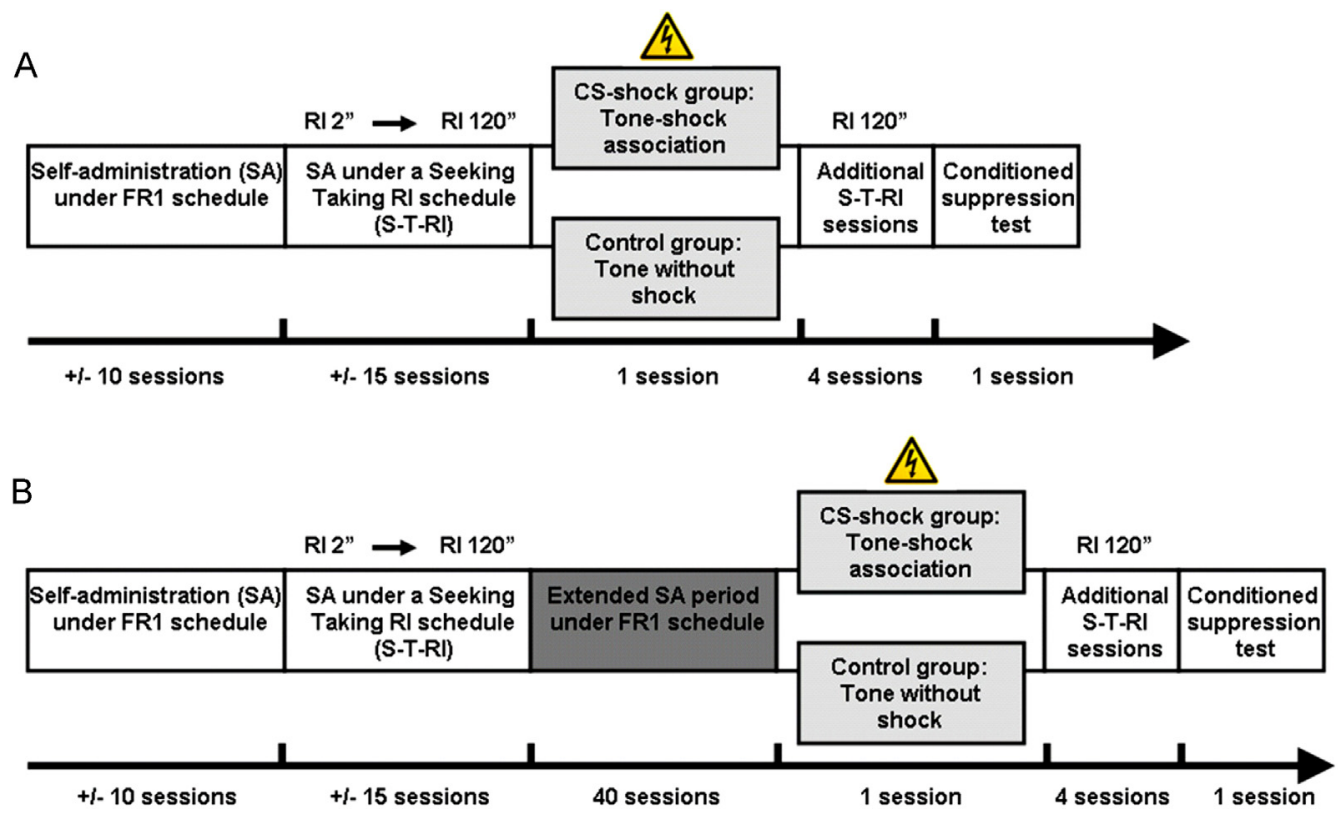

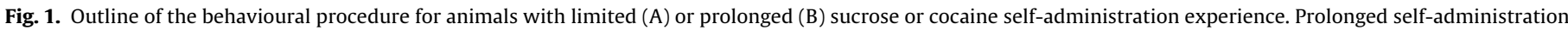
experience was achieved by allowing the animals 40 additional FR-1 self-administration sessions after responding under the ST(RI-120) schedule had stabilized. 


\section{Results}

\subsection{Experiment 1A: the effect of footshock intensity on} conditioned suppression of sucrose- or cocaine-seeking behaviour

Fig. 2 shows the effect of shock intensity on suppression ratios (Fig. 2A) and seeking latencies (Fig. 2B). There was a main effect on suppression ratio $(H(12)=13.55, p<0.05)$ as well as latency to first seeking response $(H(12)=15.56, p<0.05)$. As apparent from a lack of effect on suppression ratio and latency to first seeking response, respectively, using footshocks of $0.25 \mathrm{~mA}$ did not induce conditioned suppression of sucrose seeking (CS-shock compared to control: suppression ratio $[U=22.00$, n.s.]; latency to first response [ $U=24.50$, n.s.]). A lack of conditioned suppression of sucrose seeking was also found after conditioning with footshocks of $0.30 \mathrm{~mA}$ (CS-shock compared to control: suppression ratio $[U=28.00$, n.s. $]$; latency to first response $[U=26$, n.s.]). In contrast, conditioned suppression of sucrose seeking was observed when rats underwent CS-shock conditioning with an intensity of $0.35 \mathrm{~mA}, 0.40 \mathrm{~mA}$ and $0.45 \mathrm{~mA}$. Compared to control, conditioning with these shock intensities significantly increased the suppression ratios and latencies to first response, respectively $(0.35 \mathrm{~mA}$ : CSshock compared to control: suppression ratio [ $U=7.50, p<0.05]$; latency to first response $[U=8.00, p<0.05]$; $0.40 \mathrm{~mA}$ : CS-shock compared to control: suppression ratio $[U=0.00, p<0.05]$; latency to first response $[U=0.00, p<0.05] ; 0.45 \mathrm{~mA}$ : CS-shock compared to control: suppression ratio $[U=4.50, p<0.05]$; latency to first response $[U=6.50, p<0.05])$. Further analysis between shock intensities revealed a significant difference between the CS-shock groups conditioned with $0.30 \mathrm{~mA}$ and $0.35 \mathrm{~mA}$ shocks (suppression ratio $[U=6.50, p<0.05]$; latency to first response $[U=18.50$, $p<0.05]$ ).
In contrast to sucrose seeking, conditioned suppression of cocaine seeking was not observed in animals that underwent CSshock conditioning with footshocks of an intensity of $0.35 \mathrm{~mA}$ (CS-shock compared to control: suppression ratio [ $U=46.00$, n.s.]; latency to first response $[U=48.50$, n.s. $]$ ). However, conditioning with a footshock intensity of $0.40 \mathrm{~mA}$ and $0.45 \mathrm{~mA}$ did suppress cocaine seeking behaviour ( $0.40 \mathrm{~mA}$ : CS-shock compared to control: suppression ratio $[U=3.00, p<0.05]$; latency to first response $[U=4.00, p<0.05]$; $0.45 \mathrm{~mA}$ : CS-shock compared to control: suppression ratio $[U=2.00, p<0.05]$; latency to first response $[U=1.00$, $p<0.05]$ ). Further analysis of the data revealed a significant difference between the CS-shock groups conditioned with shock intensities of $0.35 \mathrm{~mA}$ and $0.40 \mathrm{~mA}$ (suppression ratio $[U=8.00$, $p<0.05]$; latency to first response [ $U=10.50, p<0.05]$ ). In animals conditioned with a shock intensity of $0.35 \mathrm{~mA}$, a significant difference in suppression ratio and latency to first response was found between sucrose and cocaine self-administering animals (suppression ratio $[U=6.50, p<0.05]$; latency to first response $[U=19.00$, $p<0.05]$ ).

\subsection{Experiment $1 B$ : effect of footshock quantity on conditioned suppression of sucrose-seeking behaviour}

After having determined the minimally effective footshock intensity to suppress sucrose and cocaine seeking behaviour, we next investigated whether altering the amount of footshocks influenced conditioned suppression of sucrose seeking. To that aim, rats underwent CS-shock conditioning whereby they received 10 or 20 footshocks of an intensity of $0.35 \mathrm{~mA}$ or $0.40 \mathrm{~mA}$, respectively. Fig. 3 shows the effect of shock quantity and intensity on conditioned suppression of sucrose seeking behaviour. There was a main effect on suppression ratio $(H(4)=12.87, p<0.05$; Fig. $3 \mathrm{~A}$ )

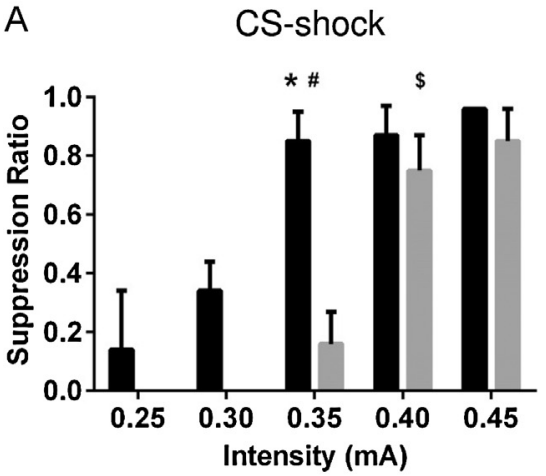

B

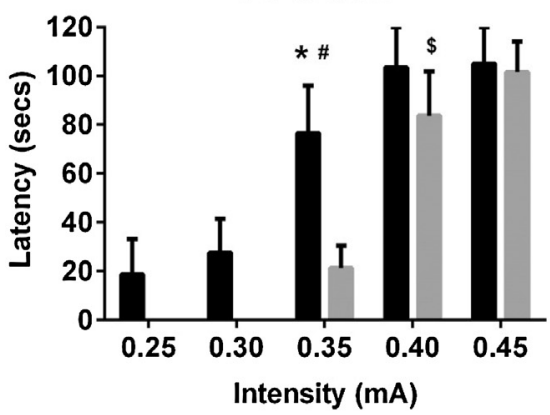

Control

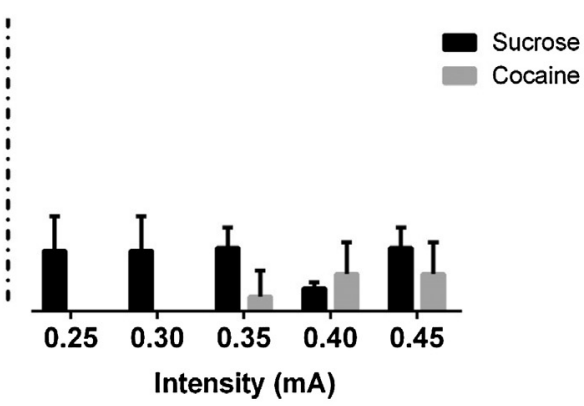

Control

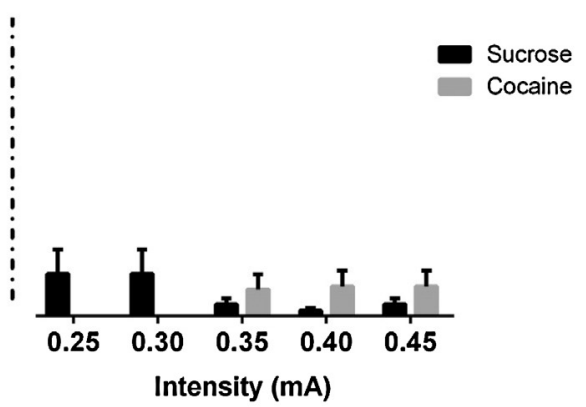

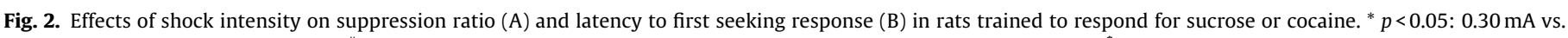

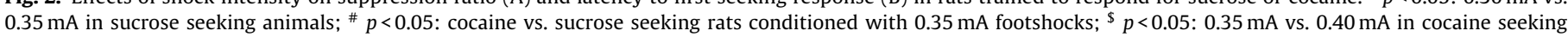

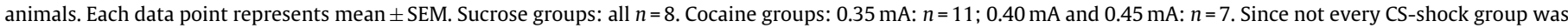

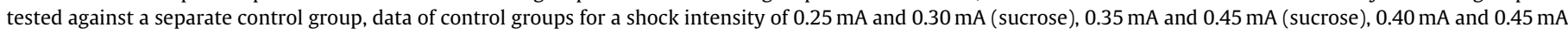
(cocaine) are the same. 
A

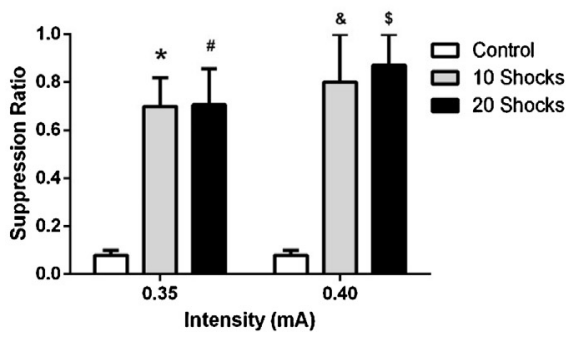

B

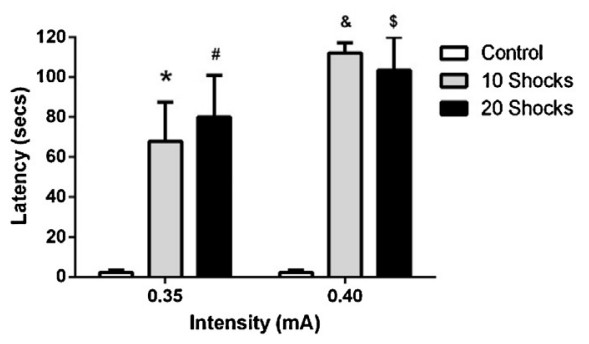

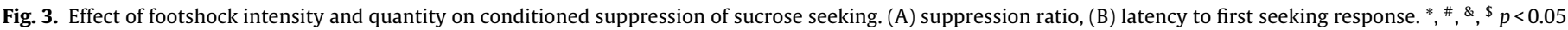

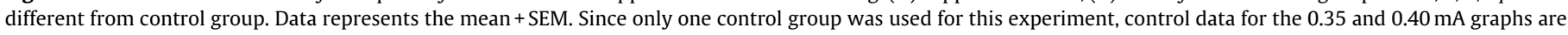
identical. All groups: $n=6$.

as well as latency to first seeking response $(H(4)=13.65, p<0.05$; Fig. 3B). Similar to experiment $1 \mathrm{~A}$, animals conditioned with 20 footshocks with an intensity of $0.35 \mathrm{~mA}$ and $0.40 \mathrm{~mA}$ showed significant conditioned suppression of sucrose seeking (CS-shock compared to control: $0.35 \mathrm{~mA}$ : suppression ratio $[U=0.00, p<0.05]$; latency to first response $[U=0.00, p<0.05]$; $0.40 \mathrm{~mA}$ : suppression ratio $[U=0.00, p<0.05]$; latency to first response $[U=0.00, p<0.05]$ ). Animals conditioned with 10 footshocks with an intensity $0.35 \mathrm{~mA}$ and $0.40 \mathrm{~mA}$ also showed conditioned suppression of sucrose seeking behaviour (CS-shock compared to control: $0.35 \mathrm{~mA}$ : suppression ratio $[U=0.00, p<0.05]$; latency to first response $[U=3.50$, $p<0.05]$; $0.40 \mathrm{~mA}$ : suppression ratio $[U=6.00, p<0.05]$; latency to first response $[U=0.00, p<0.05])$.

For both footshock intensities, changing the quantity of footshocks did not affect the suppression ratio and seeking latency in the CS-shock groups ( $0.35 \mathrm{~mA}, 10$ compared to 20 footshocks: suppression ratio $[U=18.00$, n.s.]; latency to first response $[U=16.00$, n.s.]; $0.40 \mathrm{~mA}, 10$ compared to 20 footshocks: suppression ratio $[U=12.00$, n.s.]; seeking latency $[U=11.00$, n.s. $]$ ). In addition, conditioned suppression in the CS-shock groups did not differ between the two shock intensities ( 10 footshocks, $0.35 \mathrm{~mA}$ compared to $0.40 \mathrm{~mA}$ : suppression ratio $[U=10.00$, n.s.]; latency to first response [ $U=7.00$, n.s.]; 20 footshocks, $0.35 \mathrm{~mA}$ compared to $0.40 \mathrm{~mA}$ : suppression ratio [ $U=9.00$, n.s.]; latency to first response $[U=8.00$, n.s. $])$

\subsection{Experiment 1C: effect of CS interval sequence on suppression of sucrose-seeking behaviour}

To assess the influence of differences in basal responding during a conditioned suppression test, we examined the effect of a different CS interval sequence in experiment 1C. For animals in the CS-OFF group, the test session started with a 2 min period without stimulus presentation. In contrast, for animals in the CSON group, the test session started with a 2 min CS presentation, which is similar to the procedure in experiments $1 \mathrm{~A}$ and $1 \mathrm{~B}$. There was a main effect on suppression ratio $(H(3)=9.765, p<0.05$; Fig. 4A) as well as latency to first seeking response $(H(3)=15.867$, $p<0.05$; Fig. 4B). Under either CS-interval sequence, profound conditioned suppression of sucrose seeking behaviour was apparent (ON-OFF sequence, CS-shock compared to control: suppression ratio $[U=0.00, p<0.05]$; latency to first response $[U=0.00, p<0.05]$; OFF-ON sequence, CS-shock compared to control: suppression ratio $[U=0.00, p<0.05]$; latency to first response $[U=5.50, p<0.05]$ ). No statistically significant differences in suppression ratio between the different sequences were found (CS-shock group ON-OFF sequence compared to CS-shock group OFF-ON sequence: suppression ratio $[U=11.00$, n.s. $]$; latency to first response $[U=2.00$, n.s.]).
Although these data suggest that both CS interval sequences are equally effective, seeking patterns during the conditioned suppression test differed (Fig. 4C and D). Analysis of response patterns revealed significant effects in both the CS-OFF group (group: $[F(1,30)=5.26, p<0.05]$; time: $[F(1,10)=9.56, p<0.05]$; group $\times$ time: $[F(1,30)=26.25, p<0.05] ; \operatorname{CS:~}[F(1,10)=12.65$, $p<0.05]$; group $\times \mathrm{CS}:[F(1,30)=14.57, p<0.05]$; $\mathrm{CS} \times$ time: $[F(1$, $10)=14.90$, n.s. $]$ ) and the CS-ON group (group: $[F(1,30)=7.85$, $p<0.05]$; time: $[F(1,10)=21.53, p<0.05]$; group $\times$ time: $[F(1$, $30)=14.56, p<0.05]$; $\mathrm{CS}:[F(1,10)=8.79, p<0.05]$; group $\times \operatorname{CS}:[F(1$, $30)=14.36, p<0.05]$; $C S \times$ time: $[F(1,10)=9.66$, n.s. $])$. Post-hoc analysis revealed that responding in the CS-shock animals in the CS-OFF group showed a prolonged conditioned suppression that carried over to second CS-OFF interval ( 1 st CS-ON compared to 2 nd CS-OFF: $[t(5)=2.51$, n.s. $]$ ). Moreover, seeking responses of the CSshock animals differed significantly from the control animals in the CS-OFF group during the 2nd CS-OFF interval (CS-shock compared to control: $[t(5)=2.59, p<0.05])$. In contrast, seeking responses in the CS-shock animals in the CS-ON group increased significantly from the first CS-ON to the first CS-OFF interval (1st CS-ON compared to 1st CS-OFF: $[t(5)=-6.49, p<0.05])$. Moreover, seeking responses during the first CS-OFF interval did not differ significantly between the control and CS-shock groups $[t(5)=1.10$, n.s. $]$ ).

\subsection{Experiment 1D: extinction and re-acquisition of conditioned suppression of sucrose-seeking behaviour}

In order to test the feasibility of assessing conditioned suppression within-subjects, we tested whether conditioned suppression could be extinguished and re-acquired. Fig. 5 summarizes the suppression ratios (Fig. 5A) and latency to first seeking response (Fig. 5B) of the CS-shock group in the four test sessions. There was an overall effect on suppression ratio $[\mathrm{X} 2(1)=24.00, p<0.05]$ and latency to first response $[\mathrm{X} 2(2)=11.26, p<0.05]$.

During the no CS test, the control and CS-shock groups showed no significant differences in suppression ratio $[U=10.50$, n.s. $]$ and latency to first response $[U=7.00$, n.s.]. As expected, profound conditioned suppression of seeking behaviour was observed in the CS-shock group during the conditioned suppression test (control compared to CS-shock; suppression ratio $[U=0.00, \mathrm{p}<0.05]$; latency to first response $[U=0.00, p<0.05])$. After 5 CS-extinction sessions, suppression ratio and seeking latency no longer differed between the CS-shock and control group (suppression ratio $[U=9.00$, n.s $]$; latency to first response $[U=11.00$, n.s $]$ ). However, after one CS-shock reconditioning session, a significant increase in the suppression ratio $[U=0.00, p<0.05]$ and latency to first response $[U=0.00, p<0.05]$ in the CS-shock compared to the control group was observed. 
A

CS-shock

Control
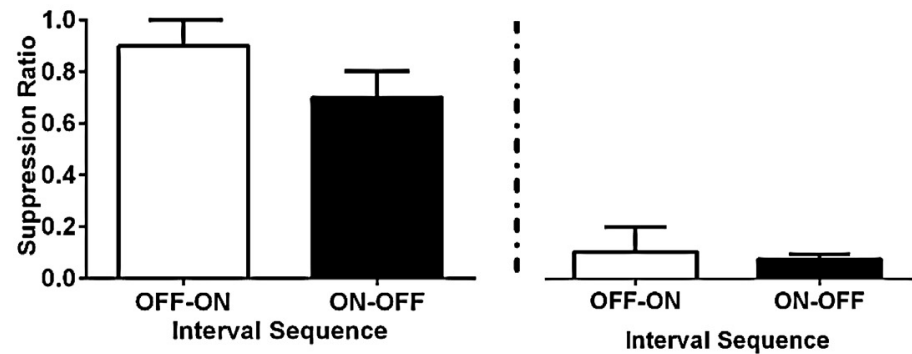

B

CS-shock

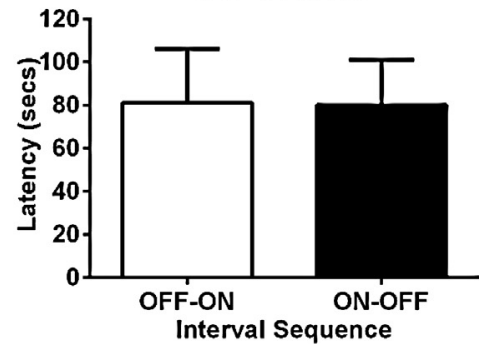

Control
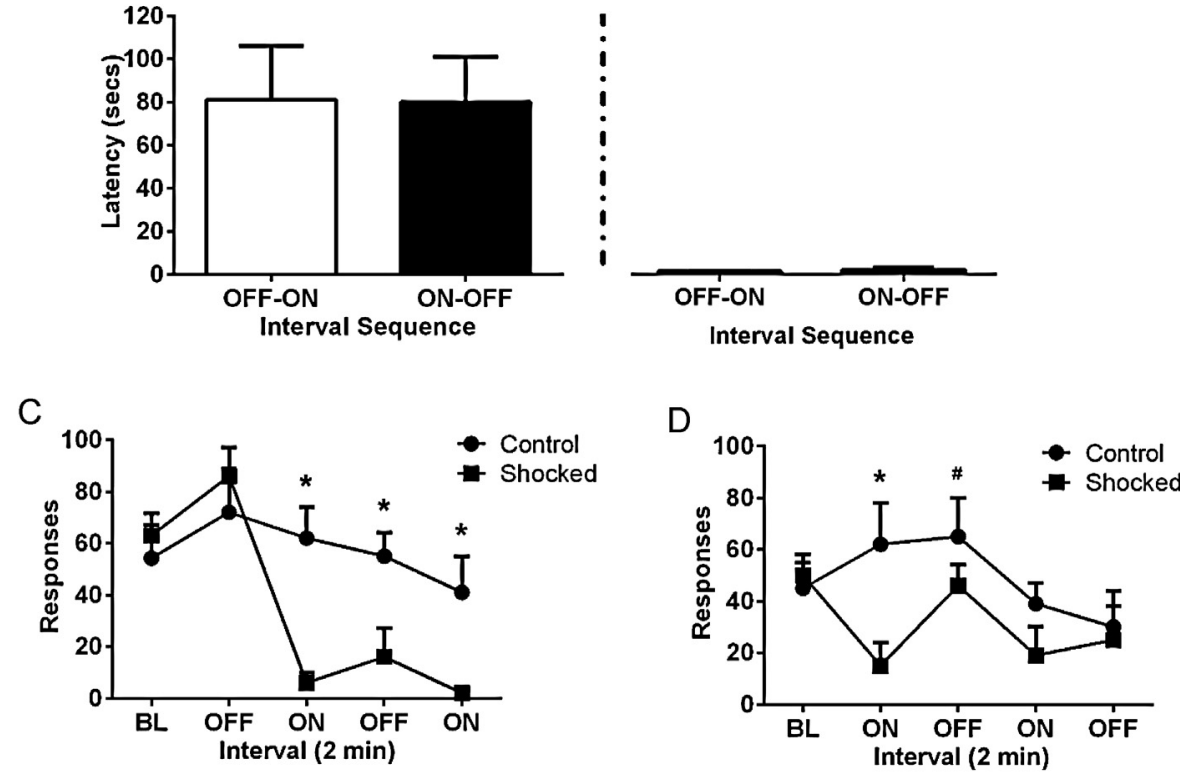

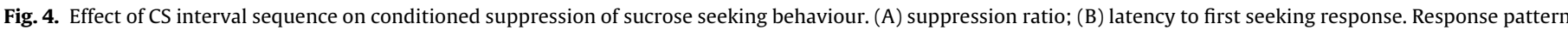

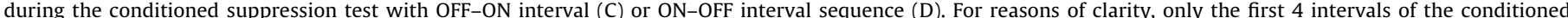

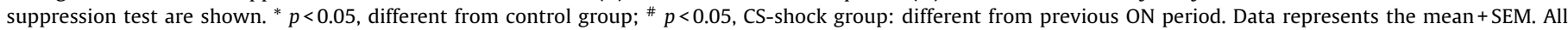
groups: $n=6$.

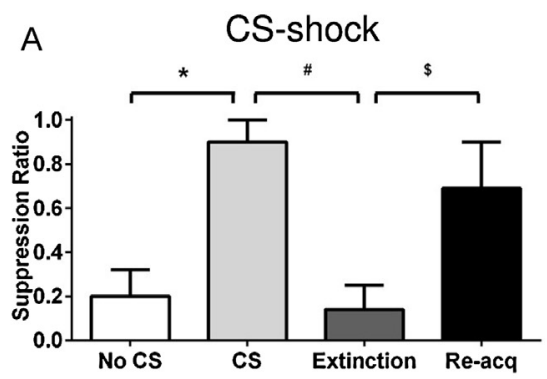

Control
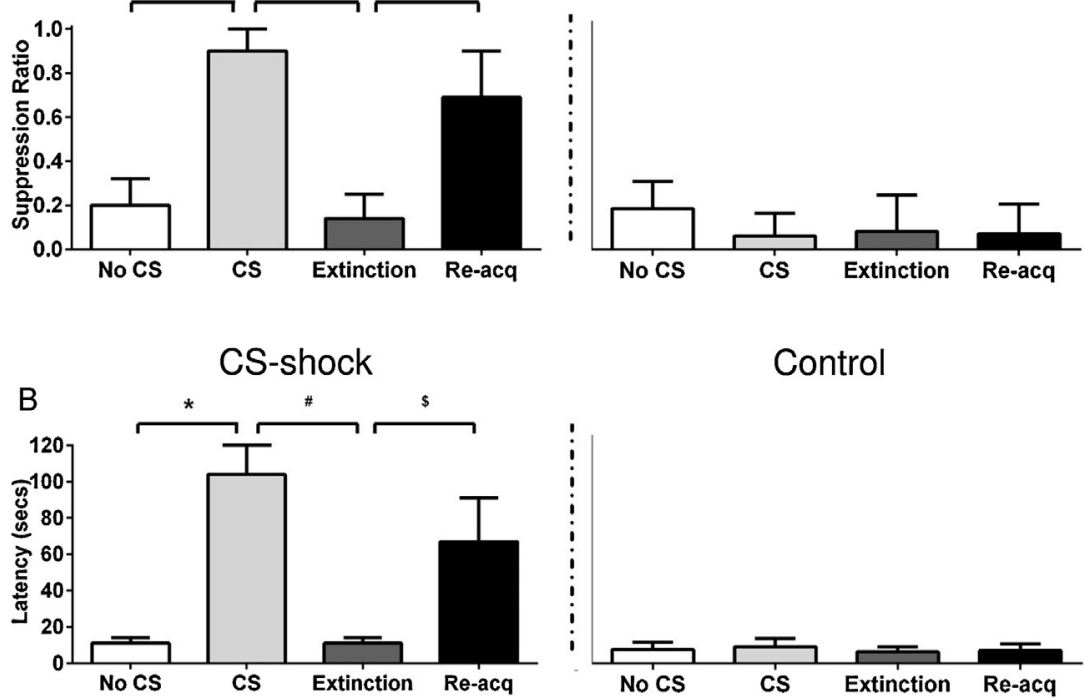

Control

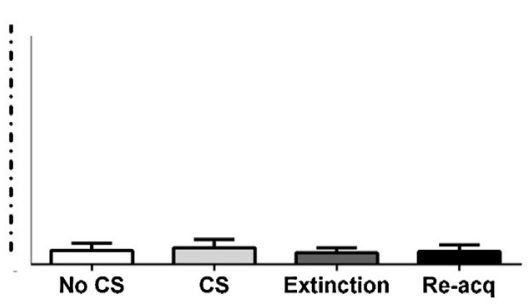

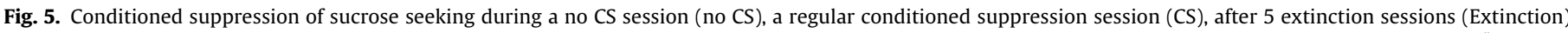

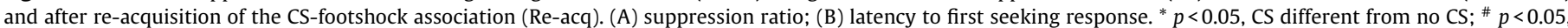
Extinction different from $\mathrm{CS} ;{ }^{\$} p<0.05$, Re-acq different from Extinction. Data represents the mean $+\mathrm{SEM}, n=6$. 
Further analysis showed a significant difference in suppression ratio $[Z=-3.08, p<0.05]$ and latency to first response $[Z=-2.96$, $p<0.05$ ] between the no CS test and the conditioned suppression test. In addition, a statistically significant difference was observed in suppression ratio $[Z=-3.08, p<0.05]$ and latency to first response $[Z=-2.52, p<0.05]$ between the conditioned suppression test and the test performed after extinction. Furthermore, suppression ratio and latency to first seeking response differed significantly between extinction and after reconditioning, respectively $[Z=-3.07, p<0.05]$, and $[Z=-2.14, p<0.05]$. Suppression ratio $[Z=-3.06$, n.s. $]$ and latency to first seeking response $[Z=-2.85, n . s$.] were not significantly different between the conditioned suppression test before extinction and the test after reconditioning.

\subsection{Summary of experiment 1: optimal conditions to investigate conditioned suppression}

The optimal experimental parameters to investigate conditioned suppression of seeking behaviour are: (1) a footshock intensity of $0.35 \mathrm{~mA}$ (sucrose) or $0.40 \mathrm{~mA}$ (cocaine). (2) CSshock conditioning with 20 footshocks. (3) Start the conditioned suppression test with a CS-ON interval. These experimental conditions were used in experiment 2 .

\subsection{Experiment 2: effect of prolonged self-administration experience on conditioned suppression of sucrose-, or cocaine-seeking behaviour}

Fig. 6 summarizes the suppression ratio (Fig. 6A) and latency to first response (Fig. 6B) of CS-shock (left panels) and control groups (right panels) after limited and extended sucrose-, or cocaine self-administration experience. Furthermore, the average response rate of the last 3 sessions under the ST(RI-120) schedule after limited and extended self-administration of sucrose (Fig. 6C) and cocaine (Fig. 6D) is shown. There was a main effect on suppression ratio $(H(7)=23.10, p<0.05)$ as well as latency to first response $(H(7)=19.58, p<0.05)$.

There was profound suppression of seeking behaviour in the CS-shock group compared to the control group after limited sucrose self-administration (suppression ratio $[U=8.00, p<0.05]$; latency to first response $[U=7.50, p<0.05]$ ). Sucrose seeking behaviour was also significantly suppressed in CS-shock group
A

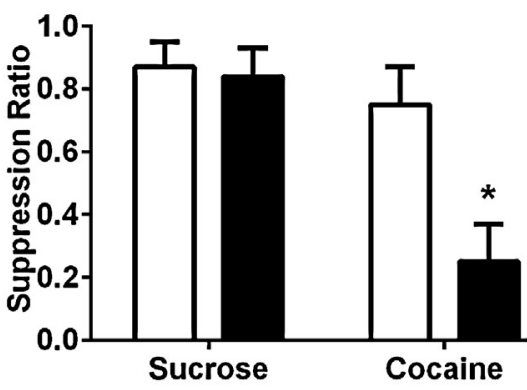

B
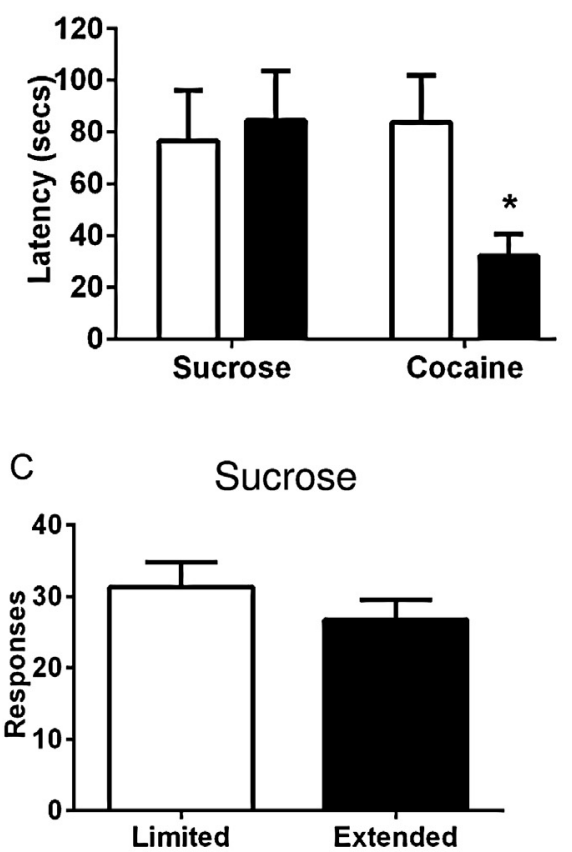

\section{Control}

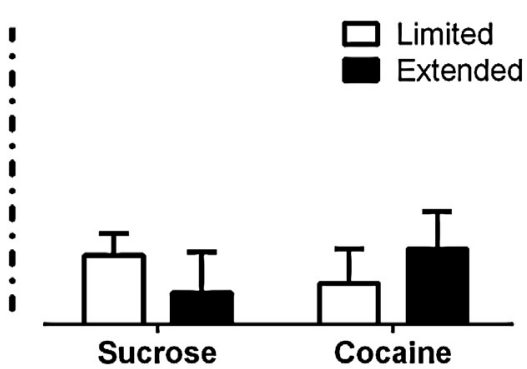

Control

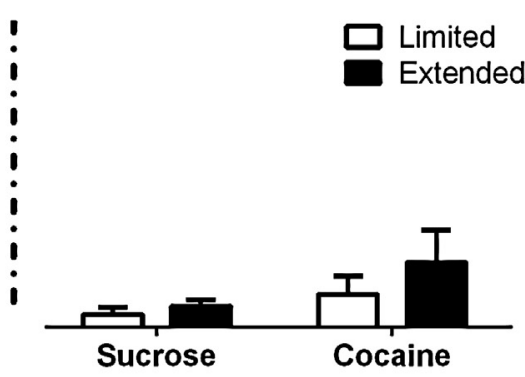

D Cocaine

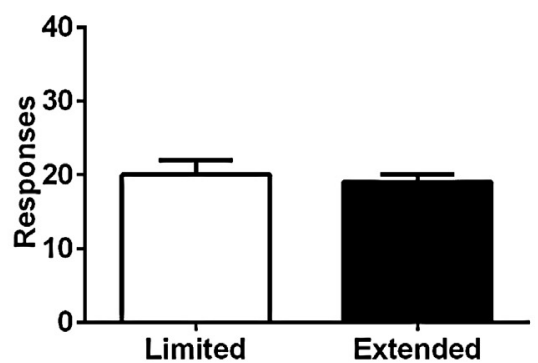

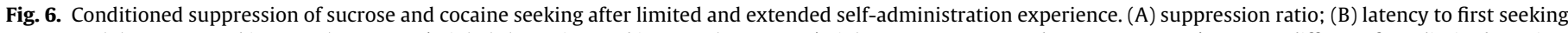

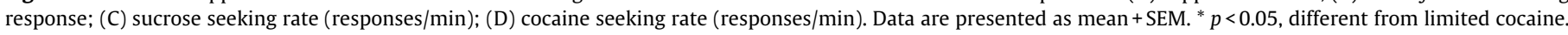
Limited sucrose: $n=8$; Extended sucrose: $n=8$; Limited cocaine: $n=7$; Extended cocaine: $n=10$. 
after extended self-administration experience (suppression ratio $[U=3.00, p<0.05]$; latency to first response $[U=7.50, p<0.05]$ ).

After a limited self-administration history, cocaine seeking behaviour was significantly suppressed in the CS-shock group compared to the control group (suppression ratio [ $U=3.00, p<0.05]$; latency to first response $[U=4.00, p<0.05]$ ). However, after extended self-administration experience, no suppression of seeking behaviour was observed in CS-shock animals compared to control group (suppression ratio $[U=32.00$, n.s.]; latency to first response $[U=44.00$, n.s. $])$. Further analysis revealed no significant difference in conditioned suppression between animals with limited and prolonged sucrose self-administration experience (CSshock groups: suppression ratio $[U=30.50$, n.s.]; latency to first response $[U=30.00$, n.s.]). In contrast, the suppression ratio and latency to first response differed significantly in the CS-shock groups between animals with limited and extended cocaine selfadministration experience (suppression ratio [ $U=11.00, p<0.05]$; latency to first response $[U=13.00, p<0.05]$ ). However, the baseline response rates in animals with limited and extended self-administration experience did not differ (sucrose-limited compared to sucrose-extended $[t(15)=-1.37$, n.s.]; cocaine-limited compared to cocaine-extended $[t(19)=-1.62$, n.s.]) (Fig. 6C-D).

\section{Discussion}

The present study was performed with two main aims. First, to optimize the conditions for conditioned suppression of sucrose and cocaine seeking. Second, to use these optimal parameters to examine the effects of conditioned suppression after limited vs. extended periods of cocaine and sucrose self-administration. We found that conditioned suppression of seeking behaviour was proportional to the intensity of the footshocks during conditioning. Thus, increased suppression of seeking was observed when animals underwent fear conditioning with a higher footshock intensity. Interestingly, a higher footshock intensity was necessary to induce conditioned suppression of cocaine seeking compared to sucrose seeking behaviour. The magnitude of conditioned suppression was not dependent upon the quantity of footshocks during conditioning. We also showed that starting the conditioned suppression test with a CS-ON interval was optimal to evoke reliable conditioned suppression. In addition, we found that conditioned suppression could be extinguished and re-acquired within subjects. With respect to our second goal, we demonstrated that the aversive CS suppressed sucrose and cocaine seeking in rats with a limited self-administration history, as well as in animals with an extended sucrose taking history. In contrast, in animals with extended cocaine experience, presentation of the footshockassociated CS no longer suppressed cocaine seeking.

We first examined the effect of different footshock intensities on the suppression of sucrose and cocaine seeking behaviour. The data showed that shock intensities of $0.25 \mathrm{~mA}$ and $0.30 \mathrm{~mA}$ did not lead to conditioned suppression of sucrose seeking behaviour. However, higher shock intensities, i.e. $0.35 \mathrm{~mA}, 0.4 \mathrm{~mA}$ and $0.45 \mathrm{~mA}$ did suppress sucrose-seeking behaviour during presence of the aversive CS. In other words, our findings indicate that the occurrence of conditioned suppression is sensitive to small differences in footshock intensity. This is consistent with literature suggesting that the magnitude of a conditioned fear response is dependent on footshock intensity (Fanselow, 1980; Morris and Bouton, 2006). Moreover, shock intensity-dependent suppression of seeking behaviour has been observed in previous studies whereby the rate of a punished response decreases as the intensity of the punishing stimulus increases (Annau and Kamin, 1961; Nader and Morgan, 2001; Simon et al., 2009). Interestingly, the present findings demonstrate that cocaine seeking behaviour is less sensitive to conditioned suppression than sucrose seeking, since higher footshock intensities were necessary to evoke suppression of cocaine seeking behaviour. Although the difference between the lowest intensity to induce conditioned suppression of cocaine seeking $(0.40 \mathrm{~mA})$ compared to sucrose seeking $(0.35 \mathrm{~mA})$ was small, the complete absence of conditioned suppression of cocaine seeking after conditioning with $0.35 \mathrm{~mA}$ footshocks does indicate that cocaine seeking is more resistant to suppression than sucrose seeking. It is not likely that cocaine seeking is less sensitive to conditioned suppression because cocaine is a more valuable reinforcer. First, in the present study, sucrose engendered higher rates of responding under baseline conditions than cocaine (Fig. 6C and D), suggesting that, if anything, sucrose is a more potent reinforcer than cocaine in these experiments. Second, recent studies have indicated that if rats are given the choice between cocaine and an ingestive reinforcer (saccharin or food), the majority of male (but not female) rats prefers the ingestive reinforcer (Lenoir et al., 2007; Cantin et al., 2010; Kerstetter et al., 2012; Perry et al., 2013). Rather, these findings resonate well with studies demonstrating that cocaine seeking is less sensitive to punishment (Jonkman et al., 2012; Pelloux et al., 2007; Vanderschuren and Everitt, 2004), and more prone to develop into inelastic behaviour (Christensen et al., 2008) than sucrose seeking. In general terms, these findings fit into a larger body of literature showing that substances of abuse and natural, food reinforcers can have differential influences on behaviour (for review, see Kearns et al., 2011).

In experiment $1 \mathrm{~B}$, we further studied the experimental parameters to optimize conditioned suppression of sucrose and cocaine seeking by analysing the effect on footshock quantity on conditioned suppression. We found that changing the quantity of footshocks (10 or 20 footshocks) had no effect on suppression of sucrose seeking behaviour. To our knowledge, there are only a limited number of studies that have directly addressed the effect of amount of tone-shock pairings on the development of conditioned fear. Thus, it has been shown that conditioning with one tone-footshock pairing evoked lower levels of conditioned freezing than five or more pairings (Maren, 1998). It is therefore possible that we may have found an effect of footshock quantity on conditioned suppression if we had used less than 10 footshocks. For the remainder of the study, we chose to use 20 shocks during conditioning, since we found in exploratory experiments that animals that had been conditioned with 20 footshocks showed more reliable conditioned suppression in terms of resistance to extinction during repeated conditioned suppression test than animals conditioned with 10 footshocks. For this reason, we did not further explore whether conditioned suppression would be absent if less than 10 footshocks were used.

In experiment $1 \mathrm{C}$, we assessed the effect of CS-interval sequence (i.e. starting the test with a CS-OFF or CS-ON interval) on conditioned suppression of sucrose seeking. We reasoned that an advantage of starting the test with a CS-OFF interval is that initial seeking rates during the test can be directly compared between the control group and the CS-shock group, providing an internal control for a comparable response rate between the groups. We observed no differences in suppression ratio and seeking latency between both CS-interval sequence approaches. However, in the rats in the CS-shock group in which the test started with a CS-OFF period, the reduction of responding during the first CS-ON period remained present during the subsequent CS-OFF period. In contrast, responding during the CS-OFF period increased compared to the first CS-ON period in the CS-shock animals in the CS-ON group (in which the test started with a 2 min presentation of the footshock-associated CS). A possible explanation for this finding is that the rats in the CS-OFF group perceived the first presentation of the footshock-associated CS as a consequence of lever pressing. In other words, after pressing the seeking lever for $2 \mathrm{~min}$, the rats were 
'punished' by the onset of the aversive CS, causing them to stop responding for the remainder of the test. Based on these data, we concluded that it is preferable to start the conditioned suppression test with a CS-ON interval.

In summary, the optimal experimental parameters to investigate conditioned suppression of sucrose and cocaine seeking are: (1) A footshock intensity of $0.35 \mathrm{~mA}$ (sucrose) or $0.40 \mathrm{~mA}$ (cocaine). (2) CS-shock conditioning with 20 footshocks. (3) Start the conditioned suppression test with a CS-ON interval.

For future experiments with a longitudinal design, it may be desirable that conditioned suppression can be measured repeatedly within-subjects. However, repeatedly testing for conditioned suppression induces a substantial reduction in the effectiveness of the conditioned aversive stimuli to suppress seeking behaviour, as a result of between-session extinction of the CS-footshock association (Limpens et al., unpublished observations). In experiment 1D, we therefore explored an approach to repeatedly measure conditioned suppression within subjects without reducing the effectiveness of the footshock-associated CS to suppress seeking. After the first conditioned suppression test, the animals underwent several CS-extinction sessions during which no footshocks were given during presentation of the tone. After extinction, suppression ratio and seeking latency were profoundly decreased. A remarkable aspect of this extinction is that although CS-footshock conditioning and CS-extinction took place in different chambers than those in which self-administration and conditioned suppression was tested, there was almost complete extinction of conditioned suppression. That is, if extinction of the CS-footshock association were completely context-dependent, then we would expect a renewal effect to take place in the self-administration context (Bouton, 1993). There are three, not mutually exclusive explanations for the absence of a renewal effect. First, extinction is not completely context-specific, since renewed responses are usually of a smaller magnitude than those before or without extinction (Bouton, 1993). Second, there may have been generalization between the contexts (i.e., chambers) in which extinction and testing took place. Third, during the test for conditioned suppression that preceded the extinction sessions, the CS but no footshocks were presented. Therefore, a certain degree of extinction may have taken place during this conditioned suppression test, so that extinction became associated with both the conditioning and testing environments. Subsequently, the rats underwent one more CS-shock conditioning session (to reinstate the tone-footshock association) followed by another conditioned suppression test. The absence of significant differences in suppression ratio and seeking latency between the pre-extinction conditioned suppression test and after re-acquisition indicates that using this approach, within-subject testing is possible without affecting the magnitude of conditioned suppression. Together, these findings show that exposure to aversive conditioned stimuli can suppress seeking behaviour, and that these suppressant effects can be weakened by extinction. After reinstating the tone-shock association with an additional CS-shock conditioning session, conditioned suppression can be re-evoked.

In experiment 2, we used the optimal experimental parameters determined in experiment 1 to confirm and extend the findings of Vanderschuren and Everitt (2004), showing that presentation of an aversive conditioned stimulus suppressed seeking behaviour in rats with limited cocaine self-administration experience, but that this no longer occurred after an extended cocaine taking history (Vanderschuren and Everitt, 2004). In the latter study, conditioned suppression was also compared between animals with an extended history of sucrose vs. cocaine self-administration, demonstrating profound suppression after prolonged sucrose but not cocaine self-administration. The absence of a group of animals with limited sucrose self-administration experience, however, leaves open the possibility that prolonged sucrose experience does alter conditioned suppression. Therefore, in the present study, we also compared animals with limited and extended sucrose selfadministration experience. Our findings show that suppression of sucrose seeking behaviour is not changed after an extended sucrose self-administration history. Consistent with the findings of Vanderschuren and Everitt (2004), we also found a dissociation between the incentive value of sucrose and cocaine (i.e. baseline seeking rates) and conditioned suppression, since baseline responding did not change, but conditioned suppression did-at least, for cocaine seeking after prolonged self-administration experience.

One may argue that the lack of suppression after extended cocaine self-administration is caused by a deficit in learning or memory, so that the animals are no longer able to encode, retrieve or express the CS-footshock association. Indeed, neurocognitive deficits in human addicts have been well documented (Garavan and Stout, 2005; Goldstein and Volkow, 2011), and drug selfadministration has been shown to evoke impairments in certain memory processes (Briand et al., 2008; Rogers et al., 2008, for review see Vanderschuren and Ahmed, 2013). However, we do not think that the lack of conditioned suppression after extended cocaine self-administration is caused by impairments in fear conditioning. First, it has been reported that a period of cocaine self-administration can also improve learning and memory in a water maze test (Del Olmo et al., 2007). Second, normal expression of conditioned fear is observed in rats after an extended cocaine self-administration history (Pelloux et al., 2007; Vanderschuren and Everitt, 2004). Based on these studies, we think that it is unlikely that impaired fear conditioning or deficits in memory are causing the resistance of cocaine seeking to presentation of the aversive CS. Moreover, it is not likely that the reduction in conditioned suppression after extended cocaine self-administration experience is the result of a change in nociceptive processing, since cocaine self-administration (for either short or long daily sessions) has been shown not to affect pain thresholds (Edwards et al., 2012).

Persistent drug use even with awareness of its deleterious consequences is a core feature of addictive behaviour (American Psychiatric Association, 2000, 2013; Leshner, 1997; O’Brien and McLellan, 1996; Volkow and Li, 2004). In recent years, several animal studies have captured inflexible drug seeking and taking behaviour (Ahmed, 2012; Deroche-Gamonet et al., 2004; Hopf et al. 2010; Lesscher et al., 2010; Pelloux et al., 2007; Seif et al., 2013; Vanderschuren and Everitt, 2004; Wolffgramm, 1991, for reviews see Lesscher and Vanderschuren, 2012; Vanderschuren and Ahmed, 2013). Interestingly, in the present study, most if not all animals became resistant to presentation of the aversive CS after prolonged cocaine self-administration. Such findings are in line with previous studies showing that with prolonged cocaine or alcohol experience, animals will become resistant to adversity (Lesscher et al., 2010; Seif et al., 2013; Vanderschuren and Everitt, 2004; Wolffgramm, 1991). It appears that the quality and contingency of presentation of the aversive stimuli determines whether resistance to punishment is observed in a subgroup of animals, or on a group level. Indeed, using footshock punishment, it has been shown that only a subgroup of animals becomes willing to endure response-contingent footshocks with prolonged cocaine or alcohol taking experience (Deroche-Gamonet et al., 2004; Pelloux et al., 2007; Seif et al., 2013, but see Jonkman et al., 2012), although all rats will give up responding if the shock intensity is high enough (Cooper et al., 2007). Together, these findings indicate that these approaches capture distinct aspects of addictive behaviour, which may rely on different neural mechanisms.

Several limitations of this study should be acknowledged. First, experiments $1 \mathrm{~B}-\mathrm{D}$ were performed in animals responding for sucrose. Although we have no reason to assume that the results of these experiments would have been substantially different in 
animals responding for cocaine, we can as yet not rule out this possibility. Second, experiments $1 \mathrm{~A}$ and 2 indicate differences between conditioned suppression of cocaine and sucrose seeking. It is tempting to interpret these differences as reflecting a difference between natural and drug reinforcers, but in order to draw this conclusion more firmly, these experiments will need to be replicated using other natural (e.g., wheel running) and drug (e.g., alcohol, heroin) reinforcers.

In summary, the present study provides an extensive parametric characterization of conditioned suppression as a method to investigate casual vs. persistent drug seeking behaviour. We have identified optimal experimental conditions to investigate conditioned suppression of sucrose and cocaine seeking. Our data show that sucrose seeking is more sensitive to conditioned suppression than cocaine seeking, and that unlike sucrose seeking, cocaine seeking becomes insensitive to conditioned suppression after prolonged self-administration experience. These findings further establish insensitivity of seeking behaviour to presentation of an aversive conditioned stimulus as a method to investigate loss of control over substance use, a defining characteristic of addictive behaviour.

\section{Role of funding source}

Funding for this study was provided by ZonMw (The Netherlands Organisation for Health Research and Development) Grant 91207006 (awarded to L.J.M.J. Vanderschuren, P. Voorn and A.B. Smit). ZonMw had no further role in study design; in the collection, analysis and interpretation of data; in the writing of the report; or in the decision to submit the paper for publication.

\section{Contributors}

JHWL, PV and LJMJV designed the study. JHWL and EHS performed the experiments and analyzed the data. JHWL, PV and LJMJV wrote the manuscript. All authors have approved the final manuscript.

\section{Conflict of interest}

The authors declare that, except for income received from their primary employers, no financial support or compensation has been received from any individual or corporate entity over the past three years for research or professional service and there are no personal financial holdings that could be perceived as constituting a potential conflict of interest.

\section{Acknowledgements}

Supported by ZonMw (The Netherlands Organisation for Health Research and Development) Grant 91207006 (awarded to L.J.M.J. Vanderschuren, P. Voorn and A.B. Smit).

\section{References}

Ahmed, S.H., 2012. The science of making drug-addicted animals. Neuroscience 211, 107-125

American Psychiatric Association, 2000. DSM-IV-R Diagnostic and Statistical Manual of Mental Disorders. American Psychiatric Association, Washington, DC, USA.

American Psychiatric Association, 2013. DSM-5 Diagnostic and Statistical Manual of Mental Disorders. American Psychiatric Association, Washington, DC, USA.

Annau, Z., Kamin, L.J., 1961. The conditioned emotional response as a function of intensity of the US. J. Comp. Physiol. Psychol. 54, 428-432.

Bouton, M.E., 1993. Context, time, and memory retrieval in the interference paradigms of Pavlovian conditioning. Psychol. Bull. 114, 80-99.

Briand, L.A., Flagel, S.B., Garcia-Fuster, M.J., Watson, S.J., Akil, H., Sarter, M., Robinson, T.E., 2008. Persistent alterations in cognitive function and prefrontal dopamine D2 receptors following extended, but not limited, access to self-administered cocaine. Neuropsychopharmacology 33, 2969-2980.
Cantin, L., Lenoir, M., Augier, E., Vanhille, N., Dubreucq, S., Serre, F., Vouillac, C. Ahmed, S.H., 2010. Cocaine is low on the value ladder of rats: possible evidence for resilience to addiction. PLoS One 5, e11592.

Christensen, C.J., Silberberg, A., Hursh, S.R., Roma, P.G., Riley, A.L., 2008. Demand for cocaine and food over time. Pharmacol. Biochem. Behav. 91, 209-216.

Cooper, A., Barnea-Ygael, N., Levy, D., Shaham, Y., Zangen, A., 2007. A conflict rat model of cue-induced relapse to cocaine seeking. Psychopharmacology 194 117-125.

Del Olmo, N., Higuera-Matas, A., Miguens, M., Garcia-Lecumberri, C., Ambrosio, E., 2007. Cocaine self-administration improves performance in a highly demanding water maze task. Psychopharmacology 195, 19-25.

Deroche-Gamonet, V., Belin, D., Piazza, P.V., 2004. Evidence for addiction-like behavior in the rat. Science 305, 1014-1017.

Dickinson, A., Wood, N., Smith, J.W., 2002. Alcohol seeking by rats: action or habit? Q. J. Exp. Psychol. B 55, 331-348.

Edwards, S., Vendruscolo, L.F., Schlosburg, J.E., Misra, K.K., Wee, S., Park, P.E., Schulteis, G., Koob, G.F., 2012. Development of mechanical hypersensitivity in rats during heroin and ethanol dependence: alleviation by $\mathrm{CRF}_{1}$ receptor antagonism. Neuropharmacology 62,1142-1151.

Fanselow, M.S., 1980. Conditioned and unconditional components of post-shock freezing. Pavlov J. Biol. Sci. 15, 177-182.

Freeman, K.B., McMaster, B.C., Roma, P.G., Woolverton, W.L., 2014. Assessment of the effects of contingent histamine injections on the reinforcing effectiveness of cocaine using behavioral economic and progressive-ratio designs. Psychopharmacology, http://dx.doi.org/10.1007/s00213-013-3396-y.

Garavan, H., Stout, J.C., 2005. Neurocognitive insights into substance abuse. Trends Cogn. Sci. 9, 195-201.

Goldstein, R.Z., Volkow, N.D., 2011. Dysfunction of the prefrontal cortex in addiction: neuroimaging findings and clinical implications. Nat. Rev. Neurosci. 12, 652-669.

Hopf, F.W., Chang, S.J., Sparta, D.R., Bowers, M.S., Bonci, A., 2010. Motivation for alcohol becomes resistant to quinine adulteration after 3 to 4 months of intermittent alcohol self-administration. Alcohol. Clin. Exp. Res. 34, 1565-1573.

Jonkman, S., Pelloux, Y., Everitt, B.J., 2012. Drug intake is sufficient, but conditioning is not necessary for the emergence of compulsive cocaine seeking after extended self-administration. Neuropsychopharmacology 37, 1612-1619.

Kearns, D.N., Weiss, S.J., Panlilio, L.V., 2002. Conditioned suppression of behavior maintained by cocaine self-administration. Drug Alcohol Depend. 65, 253-261.

Kearns, D.N., Gomez-Serrano, M.A., Tunstall, B.J., 2011. A review of preclinical research demonstrating that drug and non-drug reinforcers differentially affect behavior. Curr. Drug Abuse Rev. 4, 261-269.

Kerstetter, K.A., Ballis, M.A., Duffin-Lutgen, S., Carr, A.E., Behrens, A.M., Kippin, T.E. 2012. Sex differences in selecting between food and cocaine reinforcement are mediated by estrogen. Neuropsychopharmacology 37, 2605-2614.

Koob, G.F., Kenneth, L.G., Mason, B.J., 2009. Development of pharmacotherapies for drug addiction: a Rosetta stone approach. Nat. Rev. Drug Discov. 8, 500-515.

Lenoir, M., Serre, F., Cantin, L., Ahmed, S.H., 2007. Intense sweetness surpasses cocaine reward. PLoS One 8, e698.

Leshner, A.I., 1997. Addiction is a brain disease, and it matters. Science 278, 45-47.

Lesscher, H.M.B., van Kerkhof, L.W.M., Vanderschuren, L.J.M.J., 2010. Inflexible and indifferent alcohol drinking in male mice. Alcohol. Clin. Exp. Res. 34, 1219-1225.

Lesscher, H.M.B., Vanderschuren, L.J.M.J., 2012. Compulsive drug use and its neural substrates. Rev. Neurosci. 23, 731-745.

Maren, S., 1998. Overtraining does not mitigate contextual fear conditioning deficits produced by neurotoxic lesions of the basolateral amygdala. J. Neurosci. 18, 3088-3097.

Morris, R.W., Bouton, M.E., 2006. Effect of unconditioned stimulus magnitude on the emergence of conditioned responding. J. Exp. Psychol. Anim. Behav. Process. 32, 371-385.

Nader, M.A., Morgan, D., 2001. Effects of negative punishment contingencies on cocaine self-administration by rhesus monkeys. Behav. Pharmacol. 12, 91-99.

O’Brien, C.P., 2008. Evidence-based treatments of addiction. Philos. Trans. R. Soc. Lond. B Biol. Sci. 363, 3277-3286.

O’Brien, C.P., McLellan, A.T., 1996. Myths about the treatment of addiction. Lancet 347, 237-240.

Olmstead, M.C., Parkinson, J.A., Miles, F.J., Everitt, B.J., Dickinson, A., 2000. Cocaineseeking by rats: regulation, reinforcement and activation. Psychopharmacology $152,123-131$

Pelloux, Y., Everitt, B.J., Dickinson, A., 2007. Compulsive drug seeking by rats under punishment: effects of drug taking history. Psychopharmacology 194, 127-137.

Perry, A.N., Westenbroek, C., Becker, J.B., 2013. The development of a preference for cocaine over food identifies individual rats with addiction-like behaviors. PLoS One 8, e79465.

Pierce, R.C., O’Brien, C.P., Kenny, P.J., Vanderschuren, L.J.M.J., 2012. Rational development of addiction pharmacotherapies: successes, failures, and prospects. Cold Spring Harb. Perspect. Med. 2, a012880.

Rogers, J.L., Ghee, S., See, R.E., 2008. The neural circuitry underlying reinstatement of heroin-seeking behavior in an animal model of relapse. Neuroscience 151, 579-588.

Seif, T., Chang, S.J., Simms, J.A., Gibb, S.L., Dadgar, J., Chen, B.T., Harvey, B.K., Ron, D., Messing, R.O., Bonci, A., Hopf, F.W., 2013. Cortical activation of accumbens hyperpolarization-active NMDARs mediates aversion-resistant alcohol intake. Nat. Neurosci. 16, 1094-1100.

Simon, N.W., Gilbert, R.J., Mayse, J.D., Bizon, J.L., Setlow, B., 2009. Balancing risk and reward: a rat model of risky decision making. Neuropsychopharmacology 34, 2208-2217. 
United Nations Office on Drugs and Crime, 2012. World Drug Report 2012. United Nations, Vienna, Austria.

van den Brink, W., 2012. Evidence-based pharmacological treatment of substance use disorders and pathological gambling. Curr. Drug Abuse Rev. 5, 3-31.

Vanderschuren, L.J.M.J., Ahmed, S.H., 2013. Animal studies of addictive behavior. Cold Spring Harb. Perspect. Med. 3, a011932.

Vanderschuren, L.J.M.J., Everitt, B.J., 2004. Drug seeking becomes compulsive after prolonged cocaine self-administration. Science 305, 1017-1019.

Veeneman, M.M.J., van Ast, M., Broekhoven, M.H., Limpens, J.H.W., Vanderschuren, L.J.M.J., 2012. Seeking-taking chain schedules of cocaine and sucrose self-administration: effects of reward size, reward omission, and alphaflupenthixol. Psychopharmacology 220, 771-785.

Volkow, N.D., Li, T.K., 2004. Drug addiction: the neurobiology of behaviour gone awry. Nat. Rev. Neurosci. 5, 963-970.

Wolffgramm, J., 1991. An ethopharmacological approach to the development of drug addiction. Neurosci. Biobehav. Rev. 15, 515-519.

World Health Organization, 2004. Global Status Report On Alcohol 2004. World Health Organization, Geneva, Switzerland. 